\title{
Development Of Traditional Algorithms and Make a Hybrid Algorithm to Denoising The Color Images
}

\author{
Hanan Hamid Ali \\ hanan_hamidali@uomosul.edu.iq \\ College of Computer Sciences and Mathematic \\ University of Mosul
}

Received on 20/03/2011

Accepted on 02/11/2011

\begin{abstract}
The development in life has made the need very important for using images in many scientific fields like space, engineering and medicine. Due to the fact that such images are affected by various types of noises which produced from different sources which required some kind of filtering in order to produce a better images. The new algorithm applied in this research is obtained from merging two available methods using Matlab (2008). The first method adopts the mean filter while the second one uses the conservative smoothing filter the application done on (jpg, gif) images for (salt \& pepper) noises in different values and also (salt \& pepper, Gaussian) noises which applied at the same time on the image. The result of each method is compared with the new result outcome from the application of the new hybrid algorithm.
\end{abstract}

Keyword: smoothing, noise, conservative filter, mean filter.

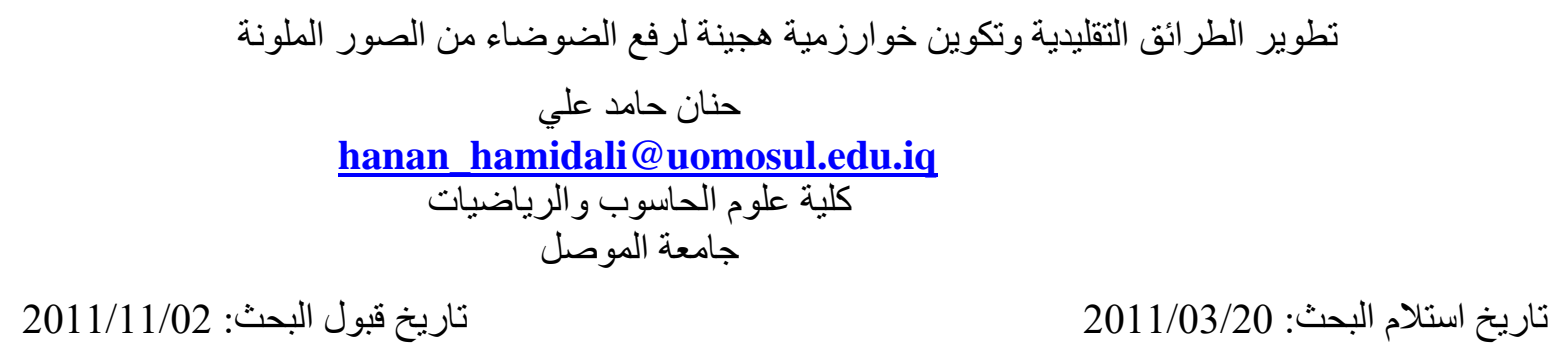

الملخص

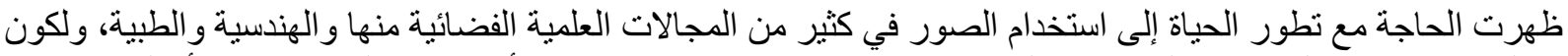

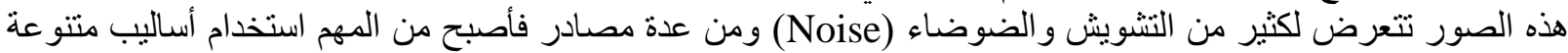

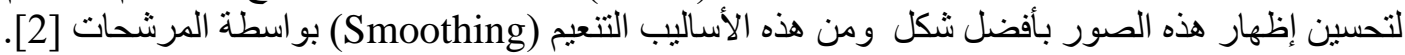

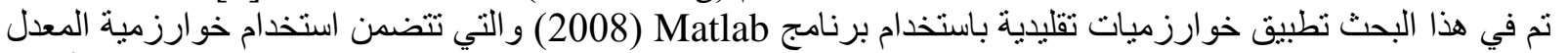

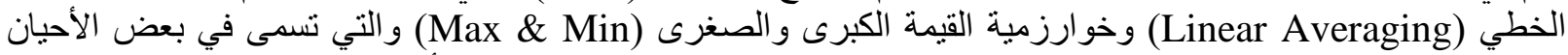

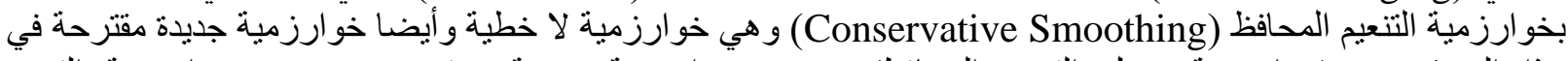

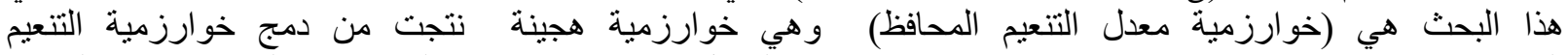

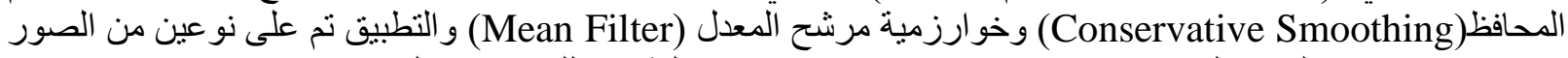

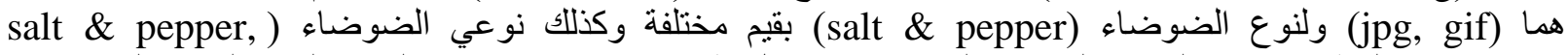
(Gaussian الهجينة الجديدة. الكلمات المفتاحية : تنعيم , ضوضاء , مرشح محافظ , مرشح المعدل . 


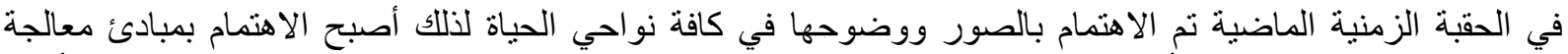

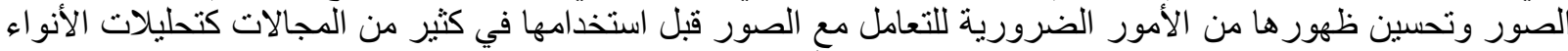

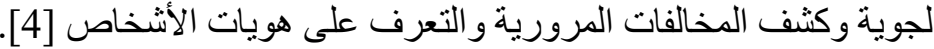

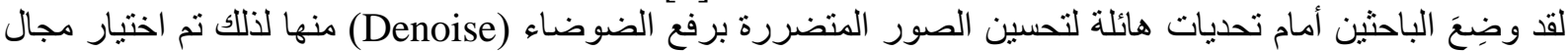
البحث للتوصل إلى خوارزمية مطورة يمكن من خلالها تحسين عرض المنات الصور الملونة بأقل ضوضاء ممكنة وبذلك تخدم الغرض من البحث.

2 هدف البحث:

يهدف البحث إلى التوصل إلى أسلوب أو طريقة تساعد على مزيد من تحسين عرض الصور الملونة وتتعيمها

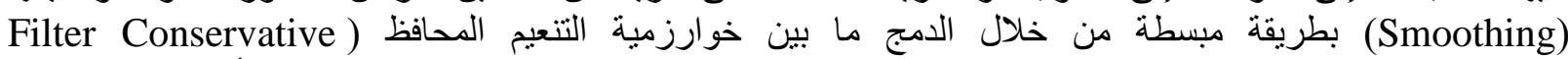
(Smoothing وخوارزمية مرشح المعدل (Mean Filter) و اقتراح خوارزمية هجينة منهما ودر اسة تأثير ذلك من خلال مقارنة الأداء لكل منهم.

3.

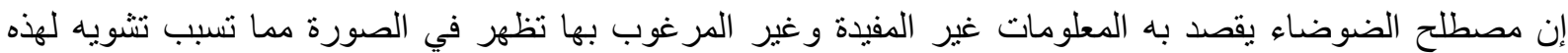

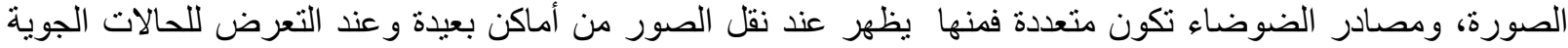

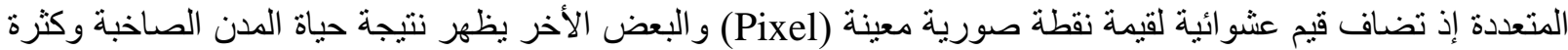

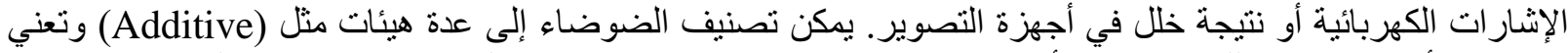

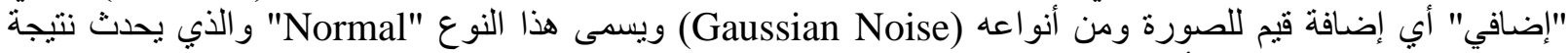

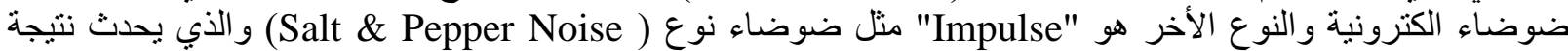

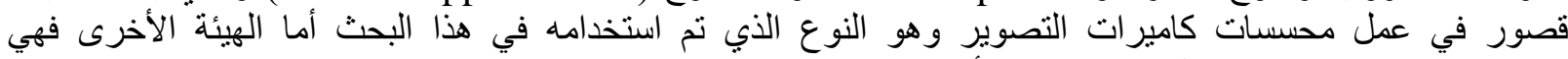
[11] وتعني "المضاعفة" ومن أنو اعه وهير (Multiplicative)

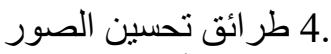
هنالك عدة أساليب متبعة لتحسين الصور تلعتمد في اغلبها على تقنية اعتماد النقاط المجاورة (neighbors) في استنتاج قيمة النقطة الجديدة ومن هذه الطر ائق طرق تقليدية وهي الطرق المتعارف عليها والئ والمنبعة علمياً ومنها طريقة المعدل الخطي

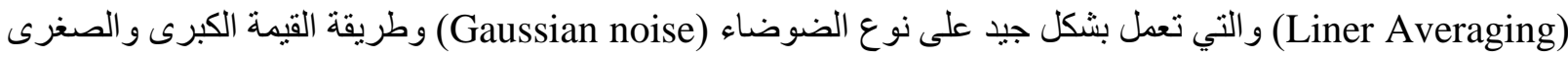
و التي تعمل بشكل جيد على نوع الضوضاء (Max \& Min)

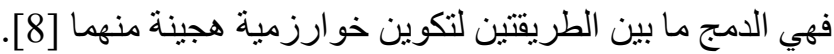

Filter Mean 4.1

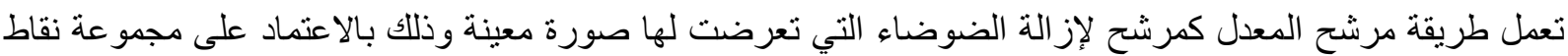

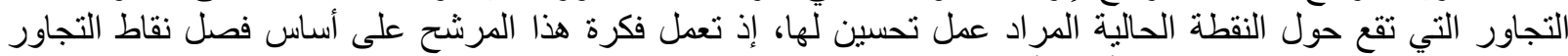

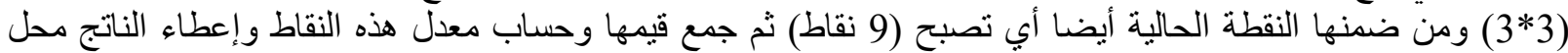

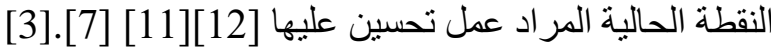

Conservative Smoothing 4.2 طريقة التنعيم المحافظ

تعمل طريقة التنعيم المحافظ كمرشح لإز الة الضوضاء عن صورة معينة وذللك بإيجاد اكبر قيمة (Max)

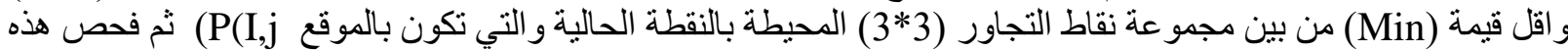

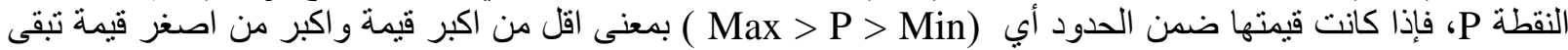

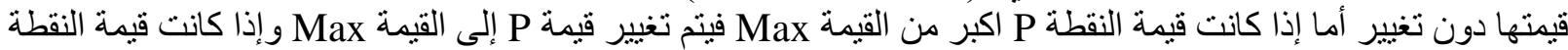

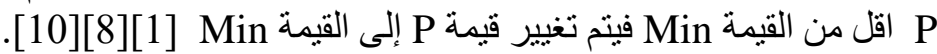

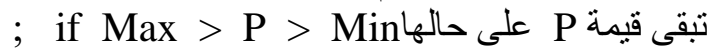

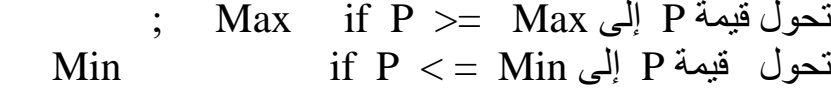

4.3 الطريقة المقترحة الهجينة (خوارزمية معدل التنعيم المحافظ)

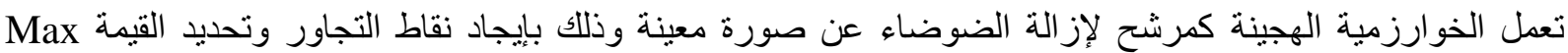

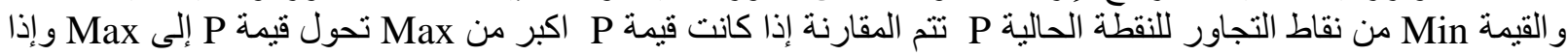




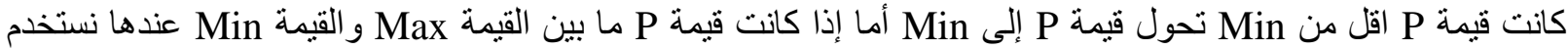

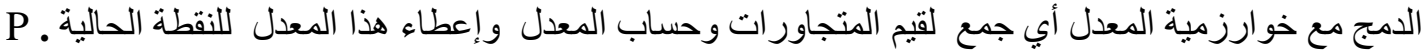

5.

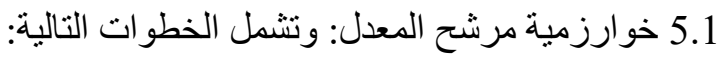

-1 قراءة الصورة وتحديد النقطة الحالية (current pixel) المر اد عمل التحسن لها كما نحدد نقاط التجاور و التي تكون بشكل حقل أو نافذة (window region) حول النقطة الحالية و هي تمثل النقاط ذات المو اقع التالية بالنسبة للنقطة الحالية ذات

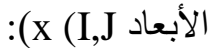
$\mathrm{x}(\mathrm{I}, \mathrm{J}-1), \mathrm{x}(\mathrm{I}, \mathrm{J}+1), \mathrm{x}(\mathrm{I}+1, \mathrm{~J}), \mathrm{x}(\mathrm{I}-1, \mathrm{~J}), \mathrm{x}(\mathrm{I}+1, \mathrm{~J}+1), \mathrm{x}(\mathrm{I}-1, \mathrm{~J}-1), \mathrm{x}(\mathrm{I}-1, \mathrm{~J}+1), \mathrm{x}(\mathrm{I}+1, \mathrm{~J}-1)$

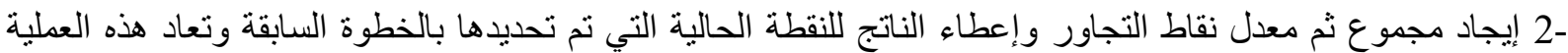
لجميع نقاط الصورة وبعدها تعرض الصورة نقطاط الناتجة. 5.2 خو ارزمية مرشح التنعيم المحافظ: وتتشمل الخطوات الفورة التالية: -1 قر اءة الصورة وتحديد نقاط التجاور للنقطة المر اد عمل التحسين لها وبعدها يتم تحديد اكبر قبمة لثدة الإضاءة من بين

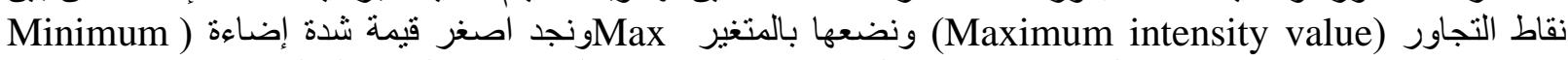

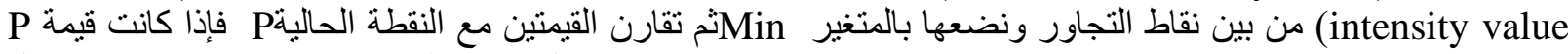

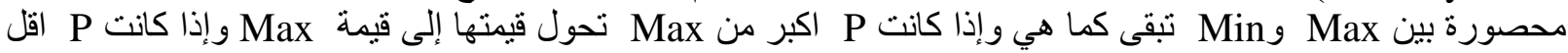

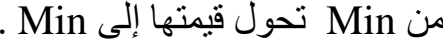
-2 نكرر العملية على جميع نقاط الصورة ثم عرض الصورة الناتجة. 5.3 الخو ارزمية المقترحة الهجينة (معدل التنعيم المحافظ):

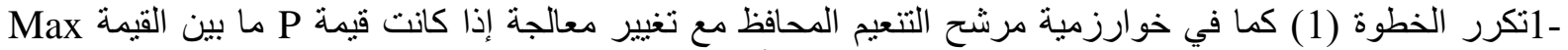

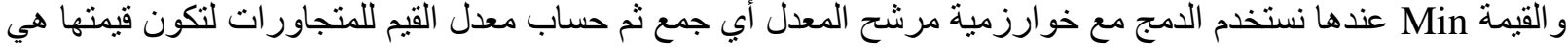

لقد تم تطبيق الخو ارزميات الثلاثة على نوعين من الصور هما (0) (jpg , gif) وبو اقع عشرة صور لكل نوع وبتطبيق قيمتين

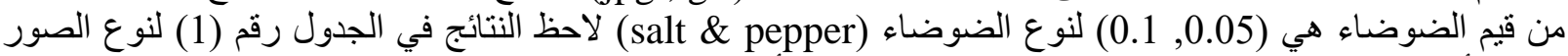

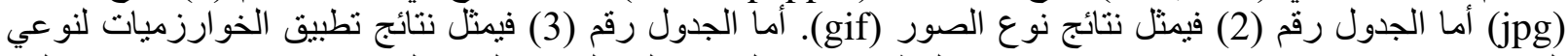

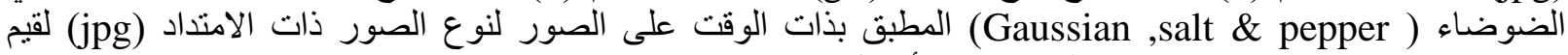

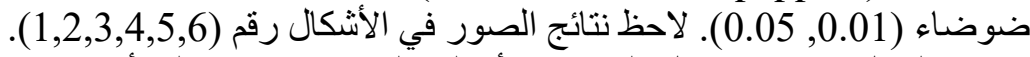

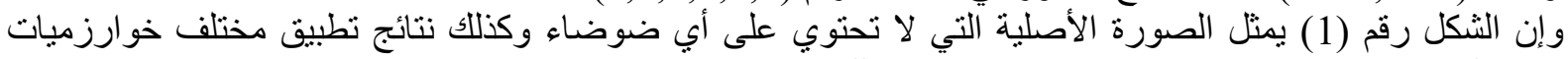

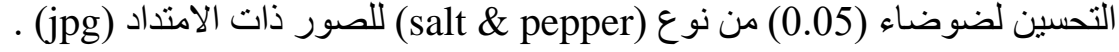




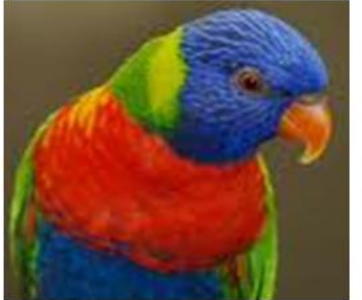

a

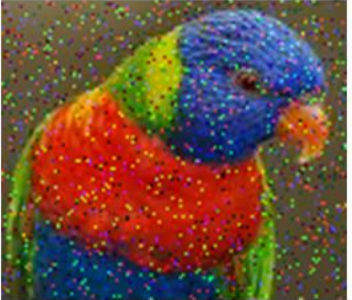

b

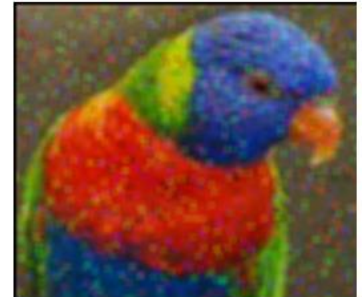

$\mathrm{c}$

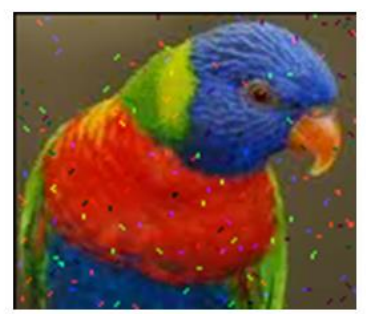

d

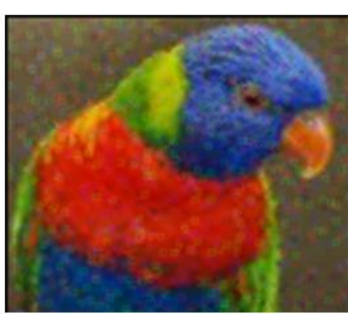

e

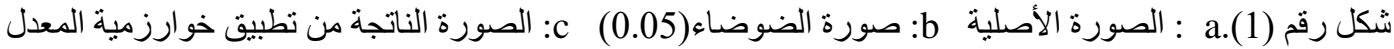
d : الصورة الناتجة من تطبيق خوارزمية المحافظ e: الصورة الناتجة من تطبيق الخوارزمية الهجينة

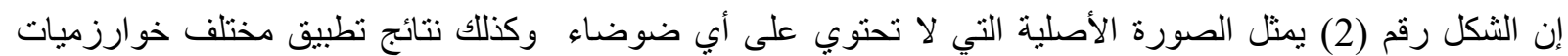

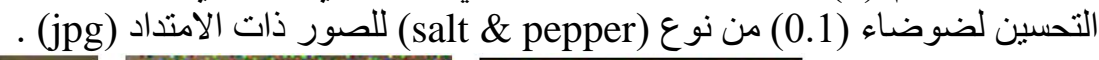

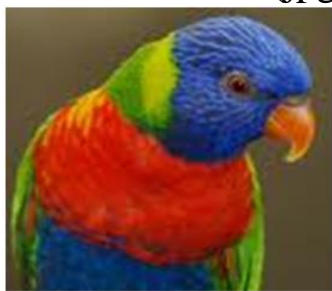

a

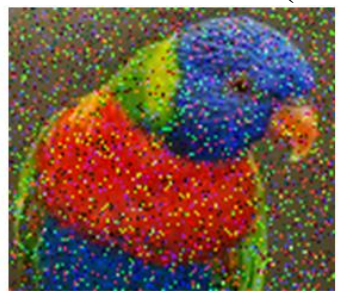

b

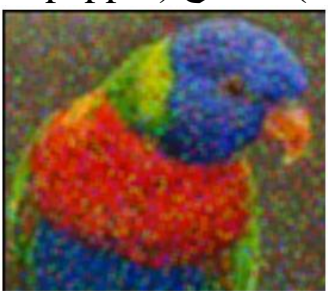

$\mathrm{c}$

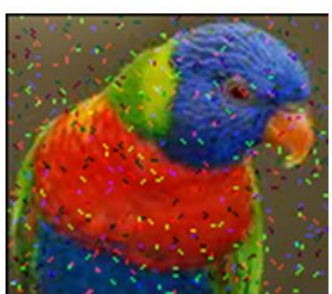

d

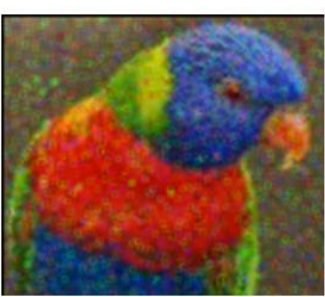

e

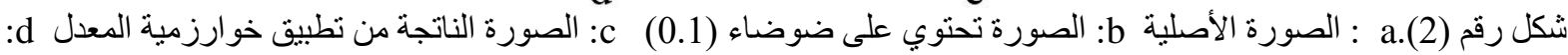
الصورة الناتجة من تطبيق خوارزمية الكحافظ e: الصورة الصورة الناتجة من تطبيق الخوارزمية الهجينة

الثكل رقم (3) يمثل الصورة الأصلية التي لا تحتوي على أي ضوضلاء وكناء وكنلك نتائج تطبيق مختلف خوارزميات التحسين لضوضاء (0.05) من نوع (3alt \& pepper) للصور ذات ذات الامتداد (Gif).

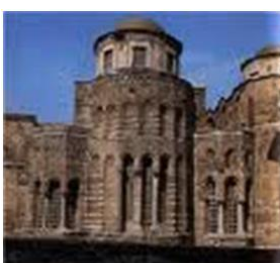

a

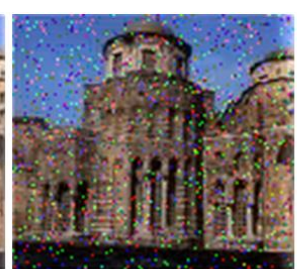

b

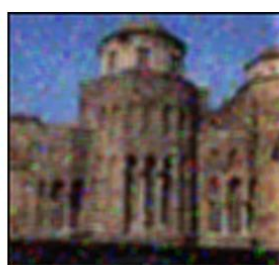

C

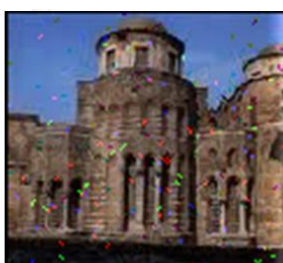

d

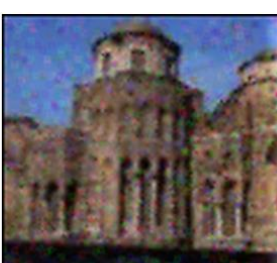

e

شكل رقم (3) : a. : الصورة الأصلية b: الصورة تحنوي على ضوضاء (0.05) c: الصورة الناتجة من تطبيق خوارزمية المعدل

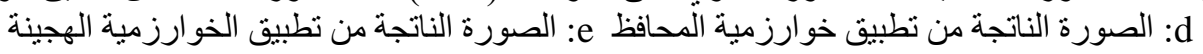


الثكل رقم (4) يمثل الصورة الأصلية التي لا تحتوي على أي ضوضلاء وكذللك نتائج تطبيق مختلف خوارزميات التحسين لضوضاء (0.1) من نوع (4alt \& pepper) للصور ذات الامتداد (Gif) .

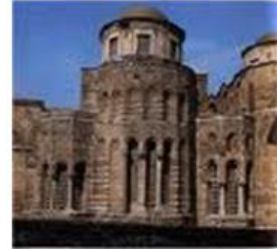

a

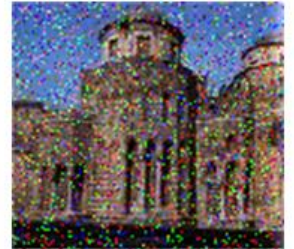

b

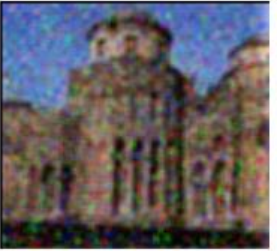

$\mathrm{c}$

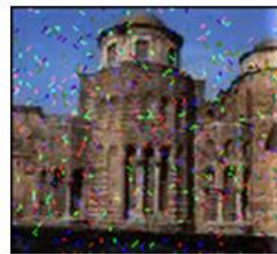

d

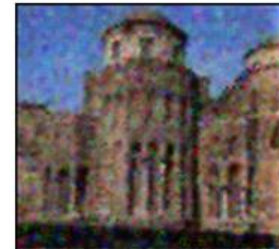

$\mathrm{e}$

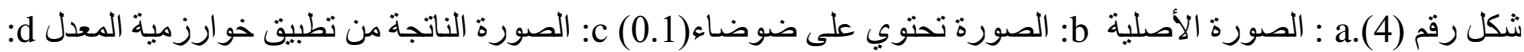

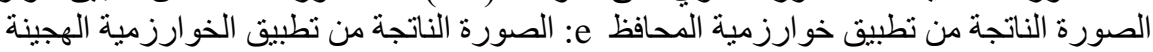

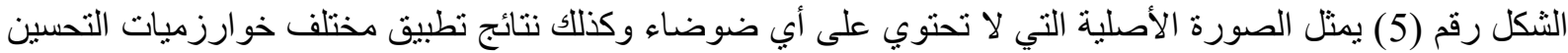
لضوضاء (0.05) من نوع (salt \& pepper) وكذللك ضوضاء من نوع (Gaussian) بقيمة (0.05) للصور ذارئ ذات الامتداد

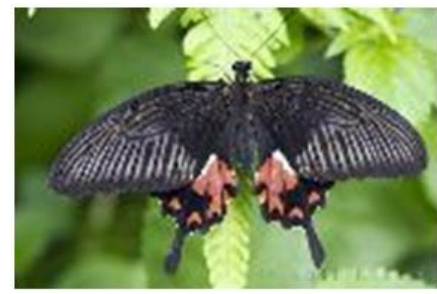

a

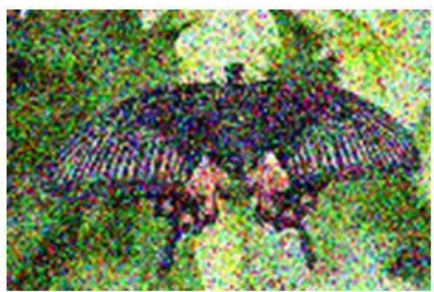

b

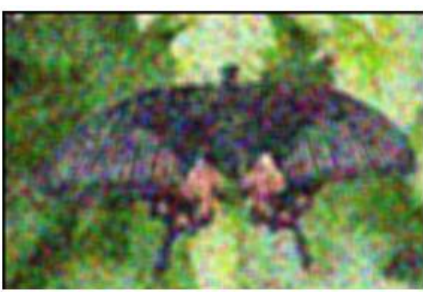

$\mathrm{c}$

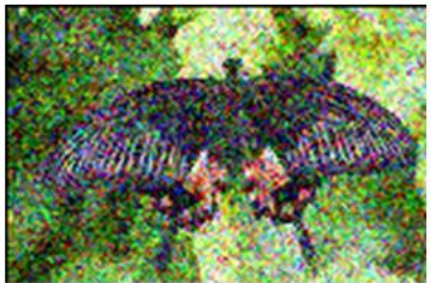

d

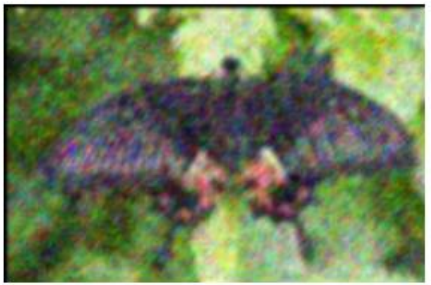

e

شكل رقم (5) : الصورة الأصلية b: صورة تحتوي على ضوضاء (0.05) للنو عين c:الصورة الناتجة من تطبيق خوارزمية المعدل

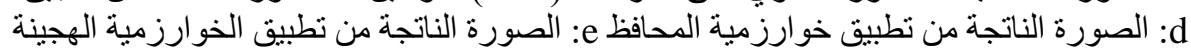

الثكل رقم (6) يمثل الصورة الأصلية التي لا تحتوي على أي ضوضاء وكذللك نتائج تطبيق مختلف خوارزميات التحسين لضوضاء (0.01) من نوع (salt \& pepper) وكذلاك ضوضاء من نوع (Gaussian) بقيمة (0.01) للصور ذات ذات الامتداد 


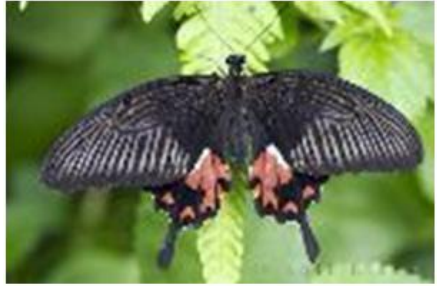

a

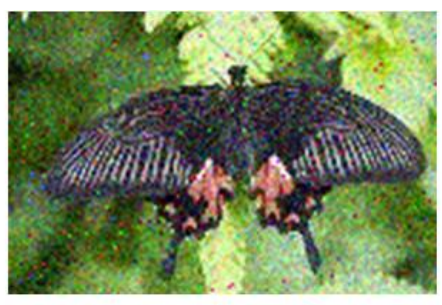

$\mathrm{b}$

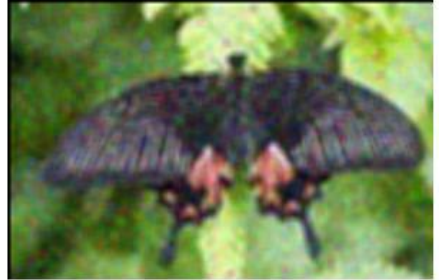

$\mathrm{C}$

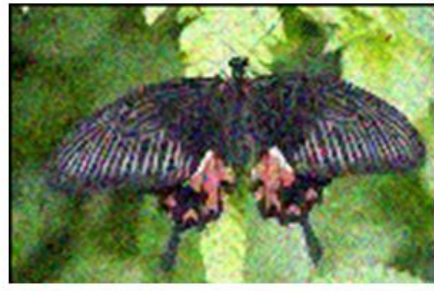

d

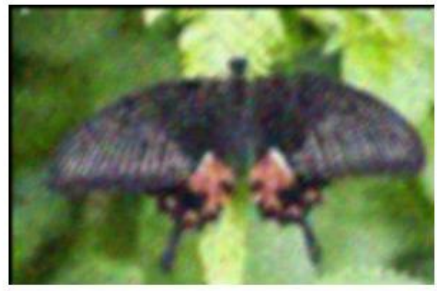

e

شكل رقم (6.) : الصورة الأصلية b : صورة تحتوي على ضوضاء (0.01) للنو عينc :الصورة الناتجة من تطبيق خوارزمية المعدل dd: الصورة الناتجة من تطبيق خوارزمية المحافظ e: الصورة الناتجة من تطبيق الخوارزمية الهجينة

لبيان مدى جودة الصور المحصلة بعد تطبيق خوارزميات التنعيم يتم استخدام مقياس error و الذي يمثل عملية مقارنة ما بين النتائج المحصلة كلٍ على حدان مع الصئل الصورة الأصلية ومن ثم المقارنة بين

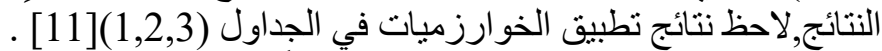
إن معادلة ان معادلة (RMSe) تمثل رياضضياً المسافة الإقليدية والتي تجد التباين بين نقاط صورتين قبل المعالجة وبعدها

$$
\begin{aligned}
& \mathrm{RMS}_{\mathrm{e}}={\sqrt{\frac{1}{\mathrm{~m} * \mathrm{n}} \sum_{\mathrm{r}=0}^{m-1} \sum_{\mathrm{c}=0}^{\mathrm{n}-1}\left[\mathrm{I}^{\prime}(\mathrm{r}, \mathrm{c})-\mathrm{I}(\mathrm{r}, \mathrm{c})\right]^{2}}}^{2} \\
& \text { والمعادلة هي] } \\
& \text { إذ تمثل I الصورة الناتجة بعد التطبيق, I سمثل الصورة الأصلية, و الصورة الكلي. }
\end{aligned}
$$

\begin{tabular}{|c|c|c|c|c|c|c|}
\hline \multicolumn{2}{|c|}{ الخو ارزمية المقترحة } & \multicolumn{2}{|c|}{ خو الرزمية التنعيم } & \multicolumn{2}{|c|}{ خوارزمية المعدل } & الخوارزمية \\
\hline 0.1 & 0.05 & 0.1 & 0.05 & 0.1 & 0.05 & مقدار الضوضوضاء \\
\hline $\begin{array}{c}20.97 \\
32\end{array}$ & $\begin{array}{c}17.69 \\
58\end{array}$ & $\begin{array}{c}30.15 \\
50\end{array}$ & $\begin{array}{c}18.77 \\
13\end{array}$ & $\begin{array}{c}22.774 \\
5\end{array}$ & $\begin{array}{c}18.85 \\
48\end{array}$ & im1 \\
\hline $\begin{array}{c}22.42 \\
86\end{array}$ & $\begin{array}{c}19.30 \\
68\end{array}$ & $\begin{array}{c}33.08 \\
30\end{array}$ & $\begin{array}{c}21.10 \\
57\end{array}$ & $\begin{array}{c}23.114 \\
6\end{array}$ & $\begin{array}{c}20.23 \\
01\end{array}$ & $\operatorname{im} 2$ \\
\hline $\begin{array}{c}18.34 \\
03\end{array}$ & $\begin{array}{c}14.71 \\
92\end{array}$ & $\begin{array}{c}27.58 \\
9\end{array}$ & $\begin{array}{c}14.72 \\
83\end{array}$ & $\begin{array}{c}19.769 \\
5\end{array}$ & $\begin{array}{c}15.56 \\
13\end{array}$ & im3 \\
\hline $\begin{array}{c}41.50 \\
05\end{array}$ & $\begin{array}{c}36.83 \\
83\end{array}$ & $\begin{array}{c}43.97 \\
29\end{array}$ & $\begin{array}{c}36.00 \\
54\end{array}$ & $\begin{array}{c}42.464 \\
7\end{array}$ & $\begin{array}{c}40.02 \\
30\end{array}$ & im4 \\
\hline $\begin{array}{c}22.37 \\
21\end{array}$ & $\begin{array}{c}18.72 \\
77\end{array}$ & $\begin{array}{c}32.10 \\
77\end{array}$ & $\begin{array}{c}20.25 \\
65\end{array}$ & $\begin{array}{c}22.936 \\
4\end{array}$ & $\begin{array}{c}19.82 \\
88\end{array}$ & im5 \\
\hline $\begin{array}{c}34.81 \\
17\end{array}$ & $\begin{array}{c}30.15 \\
16\end{array}$ & $\begin{array}{c}37.96 \\
07\end{array}$ & $\begin{array}{c}30.38 \\
94\end{array}$ & $\begin{array}{c}35.095 \\
3\end{array}$ & $\begin{array}{c}32.92 \\
48\end{array}$ & im6 \\
\hline $\begin{array}{c}32.45 \\
22\end{array}$ & $\begin{array}{c}30.41 \\
53\end{array}$ & $\begin{array}{c}37.27 \\
42\end{array}$ & $\begin{array}{c}28.91 \\
20\end{array}$ & $\begin{array}{c}33.600 \\
3\end{array}$ & $\begin{array}{c}31.60 \\
93\end{array}$ & $\operatorname{im} 7$ \\
\hline $\begin{array}{c}22.24 \\
09\end{array}$ & $\begin{array}{c}19.63 \\
73\end{array}$ & $\begin{array}{c}29.37 \\
31\end{array}$ & $\begin{array}{c}19.84 \\
19\end{array}$ & $\begin{array}{c}23.251 \\
6\end{array}$ & $\begin{array}{c}20.28 \\
20\end{array}$ & im8 \\
\hline
\end{tabular}

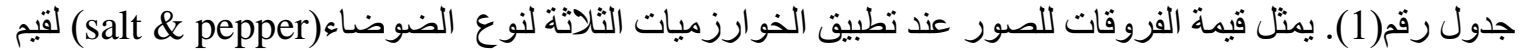

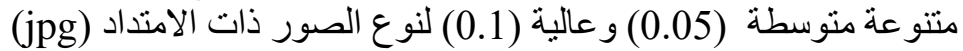




\begin{tabular}{|c|c|c|c|c|c|c|}
\hline $\mathbf{2 9 . 1 8}$ & 22.81 & 36.32 & 22.86 & 30.446 & 27.40 & im9 \\
$\mathbf{7 9}$ & 85 & 41 & 11 & 9 & 26 & \\
\hline $\mathbf{2 9 . 1 2}$ & $\mathbf{2 4 . 4 9}$ & 33.03 & 24.54 & 31.254 & 28.70 & im10 \\
$\mathbf{8 6}$ & $\mathbf{2 3}$ & 01 & 89 & 5 & 51 & \\
\hline
\end{tabular}

جدول رقم(2). يمثل قيمة الفروقات للصور عند تطبيق الخوارزميات الثلاثة لنوع الضوضاء (salt \& pepper) لقيم منتو عة منوسطة(0.05) و عالية (0.1) لنوع الصور ذات التونة الامتداد (Gif)

\begin{tabular}{|c|c|c|c|c|c|c|}
\hline \multicolumn{2}{|c|}{ الخوارزمية المقترحة } & \multicolumn{2}{|c|}{ خوارزمية التتعيم } & \multicolumn{2}{|c|}{ خوارزمية المعدل } & الخو ارزمية \\
\hline 0.1 & 0.05 & 0.1 & 0.05 & 0.1 & 0.05 & مقدار الضـوضاء \\
\hline $\begin{array}{c}25.50 \\
09\end{array}$ & $\begin{array}{c}20.91 \\
08\end{array}$ & $\begin{array}{c}30.66 \\
49\end{array}$ & $\begin{array}{c}21.28 \\
51\end{array}$ & $\begin{array}{c}27.27 \\
93\end{array}$ & $\begin{array}{c}24.92 \\
59\end{array}$ & im1 \\
\hline $\begin{array}{c}32.73 \\
38\end{array}$ & $\begin{array}{c}30.60 \\
79\end{array}$ & $\begin{array}{c}37.68 \\
49\end{array}$ & $\begin{array}{c}29.94 \\
29\end{array}$ & $\begin{array}{c}33.88 \\
40\end{array}$ & $\begin{array}{c}31.73 \\
29\end{array}$ & $\operatorname{im2}$ \\
\hline $\begin{array}{c}22.38 \\
60\end{array}$ & $\begin{array}{c}16.02 \\
78\end{array}$ & $\begin{array}{c}31.57 \\
73\end{array}$ & $\begin{array}{c}16.11 \\
38\end{array}$ & $\begin{array}{c}24.92 \\
54\end{array}$ & $\begin{array}{c}19.18 \\
36\end{array}$ & $\operatorname{im} 3$ \\
\hline $\begin{array}{c}18.34 \\
43\end{array}$ & $\begin{array}{c}12.99 \\
48\end{array}$ & $\begin{array}{c}28.88 \\
18\end{array}$ & $\begin{array}{c}15.81 \\
59\end{array}$ & $\begin{array}{c}19.59 \\
65\end{array}$ & $\begin{array}{c}13.85 \\
19\end{array}$ & im4 \\
\hline $\begin{array}{c}37.72 \\
74\end{array}$ & $\begin{array}{c}35.12 \\
76\end{array}$ & $\begin{array}{c}41.41 \\
42\end{array}$ & $\begin{array}{c}32.53 \\
95\end{array}$ & $\begin{array}{c}39.63 \\
66\end{array}$ & $\begin{array}{c}37.39 \\
85\end{array}$ & im5 \\
\hline $\begin{array}{c}31.18 \\
75\end{array}$ & $\begin{array}{c}27.21 \\
99\end{array}$ & $\begin{array}{c}35.12 \\
26\end{array}$ & $\begin{array}{c}27.30 \\
75\end{array}$ & $\begin{array}{c}32.94 \\
27\end{array}$ & $\begin{array}{c}30.06 \\
61\end{array}$ & im6 \\
\hline $\begin{array}{c}40.11 \\
64\end{array}$ & $\begin{array}{c}37.53 \\
43\end{array}$ & $\begin{array}{c}46.57 \\
21\end{array}$ & $\begin{array}{c}38.37 \\
07\end{array}$ & $\begin{array}{c}41.13 \\
56\end{array}$ & $\begin{array}{c}37.95 \\
03\end{array}$ & im7 \\
\hline $\begin{array}{c}26.26 \\
05\end{array}$ & $\begin{array}{c}21.43 \\
07\end{array}$ & $\begin{array}{c}32.08 \\
97\end{array}$ & $\begin{array}{c}22.08 \\
86\end{array}$ & $\begin{array}{c}27.92 \\
30\end{array}$ & $\begin{array}{c}25.30 \\
89\end{array}$ & im8 \\
\hline $\begin{array}{c}43.51 \\
26\end{array}$ & $\begin{array}{c}38.15 \\
04\end{array}$ & $\begin{array}{c}46.93 \\
51\end{array}$ & $\begin{array}{c}39.13 \\
39\end{array}$ & $\begin{array}{c}43.94 \\
26\end{array}$ & $\begin{array}{c}40.49 \\
31\end{array}$ & im9 \\
\hline $\begin{array}{c}41.18 \\
52\end{array}$ & $\begin{array}{c}38.29 \\
03\end{array}$ & $\begin{array}{c}46.97 \\
29\end{array}$ & $\begin{array}{c}37.76 \\
34\end{array}$ & $\begin{array}{c}42.30 \\
49\end{array}$ & $\begin{array}{c}39.24 \\
69\end{array}$ & $\operatorname{im} 10$ \\
\hline
\end{tabular}

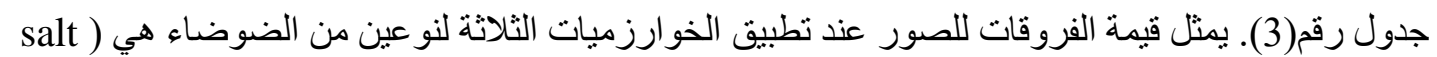

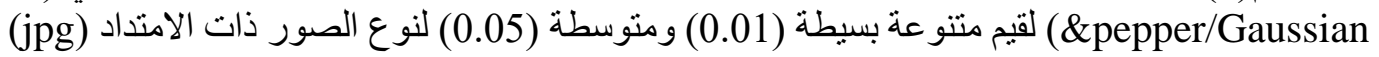

\begin{tabular}{|c|c|c|c|c|c|c|}
\hline \multicolumn{2}{|c|}{ الخوارزمية المقترحة } & \multicolumn{2}{|c|}{ خوارزمية التنعيم } & \multicolumn{2}{|c|}{ خوارزمية المعدل } & الخو ارزمية \\
\hline 0.05 & 0.01 & 0.05 & 0.01 & 0.05 & 0.01 & مقدار الضوضاف \\
\hline $\begin{array}{c}24.344 \\
3\end{array}$ & $\begin{array}{c}18.09 \\
65\end{array}$ & $\begin{array}{c}49.77 \\
58\end{array}$ & $\begin{array}{c}25.37 \\
22\end{array}$ & $\begin{array}{c}26.17 \\
64\end{array}$ & $\begin{array}{c}18.24 \\
21\end{array}$ & im1 \\
\hline $\begin{array}{c}25.101 \\
9\end{array}$ & $\begin{array}{c}18.11 \\
32\end{array}$ & $\begin{array}{c}47.80 \\
11\end{array}$ & $\begin{array}{c}25.58 \\
94\end{array}$ & $\begin{array}{c}26.69 \\
43\end{array}$ & $\begin{array}{c}18.61 \\
65\end{array}$ & $\operatorname{im} 2$ \\
\hline $\begin{array}{c}21.790 \\
2\end{array}$ & $\begin{array}{c}13.58 \\
72\end{array}$ & $\begin{array}{c}46.25 \\
39\end{array}$ & $\begin{array}{c}21.95 \\
98\end{array}$ & $\begin{array}{c}24.21 \\
19\end{array}$ & $\begin{array}{c}14.05 \\
97\end{array}$ & im3 \\
\hline $\begin{array}{c}45.962 \\
7\end{array}$ & $\begin{array}{c}41.27 \\
83\end{array}$ & $\begin{array}{c}54.52 \\
12\end{array}$ & $\begin{array}{c}36.88 \\
76\end{array}$ & $\begin{array}{c}46.46 \\
66\end{array}$ & $\begin{array}{c}39.49 \\
66\end{array}$ & im4 \\
\hline $\begin{array}{c}25.890 \\
6\end{array}$ & $\begin{array}{c}18.23 \\
01\end{array}$ & $\begin{array}{c}47.64 \\
94\end{array}$ & $\begin{array}{c}24.45 \\
67\end{array}$ & $\begin{array}{c}27.21 \\
77\end{array}$ & $\begin{array}{c}18.46 \\
70\end{array}$ & $\operatorname{im5}$ \\
\hline $\begin{array}{c}38.279 \\
0\end{array}$ & $\begin{array}{c}31.09 \\
19\end{array}$ & $\begin{array}{c}51.82 \\
92\end{array}$ & $\begin{array}{c}31.69 \\
45\end{array}$ & $\begin{array}{c}38.77 \\
57\end{array}$ & $\begin{array}{c}32.01 \\
42\end{array}$ & im6 \\
\hline 36.381 & 31.10 & 52.48 & 33.09 & 36.69 & 31.15 & im7 \\
\hline
\end{tabular}




\begin{tabular}{|c|c|c|c|c|c|c|}
\hline $\mathbf{1}$ & $\mathbf{6 4}$ & 99 & 39 & 48 & 00 & \\
\hline $\mathbf{2 5 . 6 5 3}$ & 20.10 & 50.30 & 26.47 & 27.15 & $\mathbf{1 9 . 7 4}$ & im8 \\
$\mathbf{0}$ & 42 & 09 & 22 & 02 & $\mathbf{5 0}$ & \\
\hline $\mathbf{3 3 . 7 4 4}$ & 25.24 & 47.12 & $\mathbf{2 5 . 2 0}$ & 36.22 & 26.67 & im9 \\
$\mathbf{4}$ & 00 & 84 & $\mathbf{7 6}$ & 05 & 69 & \\
\hline $\mathbf{3 3 . 3 0 6}$ & $\mathbf{2 8 . 4 3}$ & 51.18 & 28.59 & 33.52 & 28.44 & im10 \\
$\mathbf{4}$ & $\mathbf{4 2}$ & 11 & 10 & 23 & 70 & \\
\hline
\end{tabular}

وللتأكد من جودة نتائج الصور المحصلة يمكن استخدام مقياس آخر هو (NCC) وهو مقياس معامل الارتباط (Normalization Correlation Coefficient)

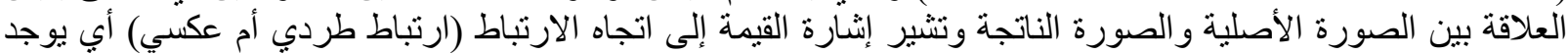

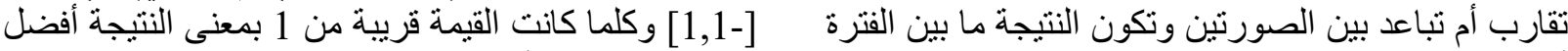

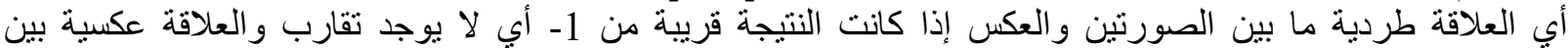

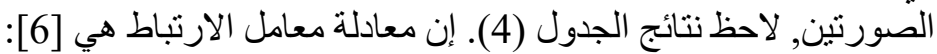

$$
N C C=\sum_{x, y} P(x, y) P^{-}(x, y) / \sum_{x, y} P^{2}(x, y)
$$

إذ تمثل p(x,y) النقطة الأصلية , (x,y) النقطة المحسنة.

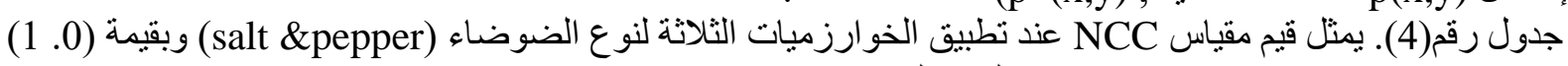

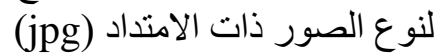

\begin{tabular}{|c|c|c|c|}
\hline الخوارزمية الهجينة & خوارزمية التنعيم & خوارزمية المعدل & الخوارزمية \\
\hline 0.9270 & 0.8675 & 0.9180 & im1 \\
\hline 0.9578 & 0.9196 & 0.9525 & $\operatorname{im} 2$ \\
\hline 0.9630 & 0.9146 & 0.9568 & $\operatorname{im3}$ \\
\hline 0.8585 & 0.8584 & 0.8542 & im4 \\
\hline 0.9198 & 0.8815 & 0.9119 & im5 \\
\hline 0.9068 & 0.8957 & 0.9005 & im6 \\
\hline 0.8902 & 0.8790 & 0.8849 & im7 \\
\hline 0.8256 & 0.7735 & 0.7995 & im8 \\
\hline 0.9500 & 0.9268 & 0.9444 & im9 \\
\hline 0.8757 & 0.8598 & 0.8559 & im10 \\
\hline
\end{tabular}

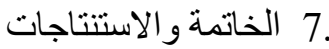

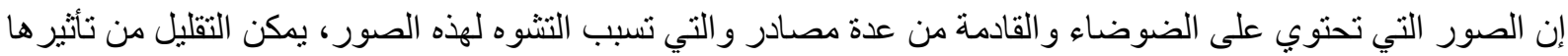

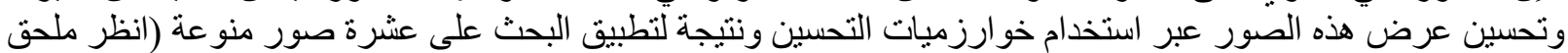

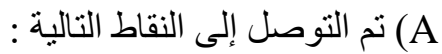

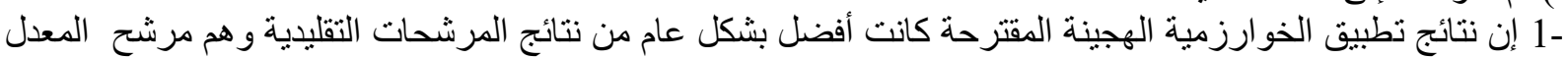

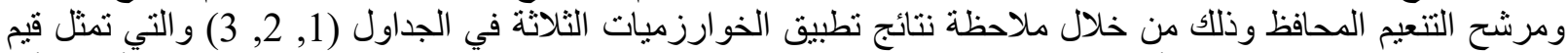

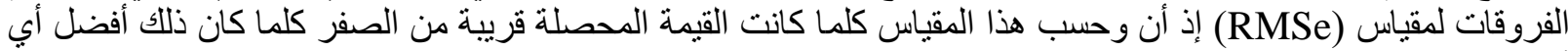
أن جودة الصورة المحصلة جيدة وقليلة التشويله -2 إن تطبيق الخوارزميات على نو عين و اسعي الانتشار من أنواع الصونة الصور و هما (jpg , gif ) لم يؤثر على نتائج التطبيق

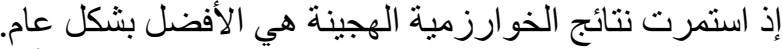

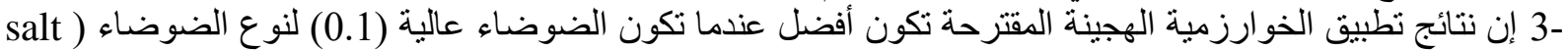
(\& pepper

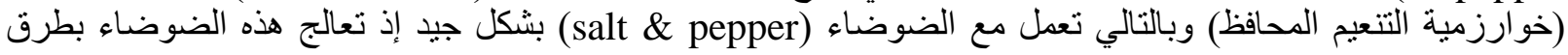


مرشحات اللاخطية أي يمكن إزالة هذا النوع من الضوضاء, أما عندما تكون الضوضاء منوسطة (0.05) أيضا تعمل

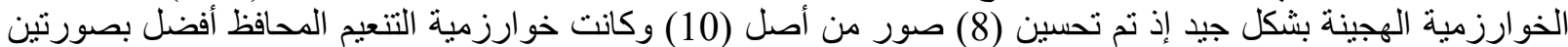

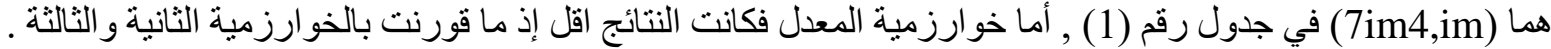

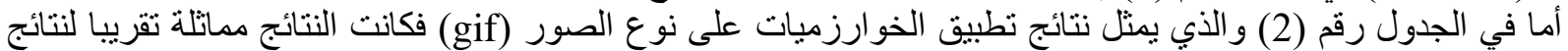

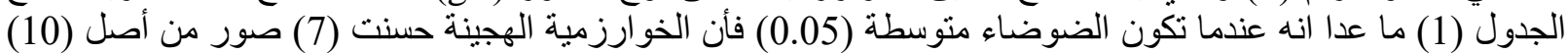

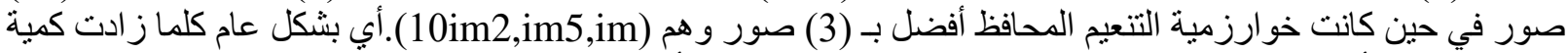

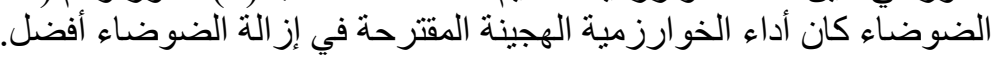

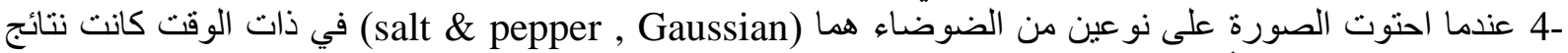

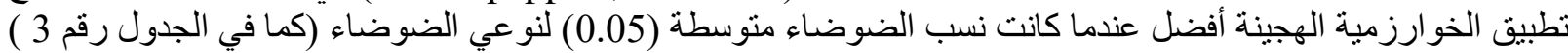

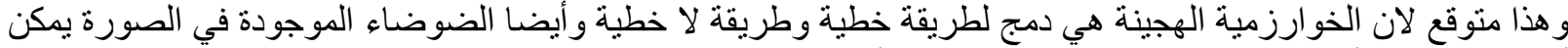

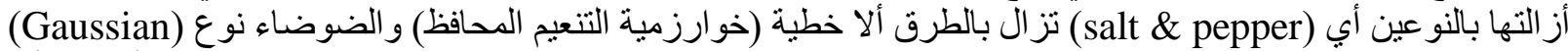

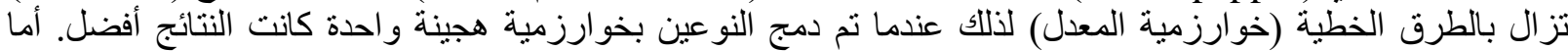

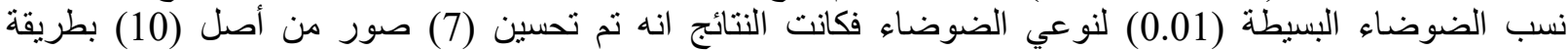

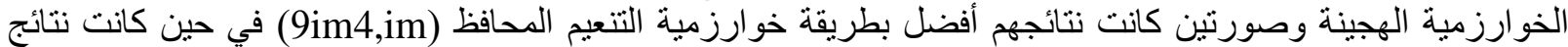

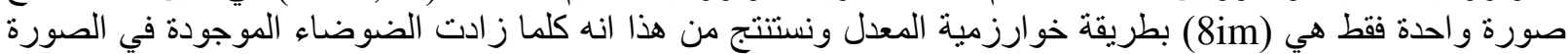

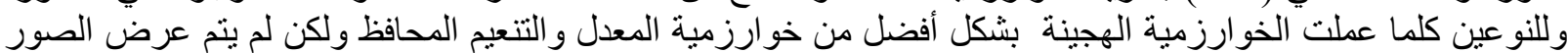

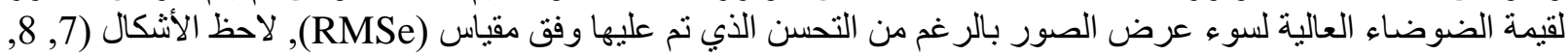

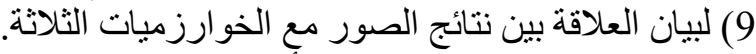

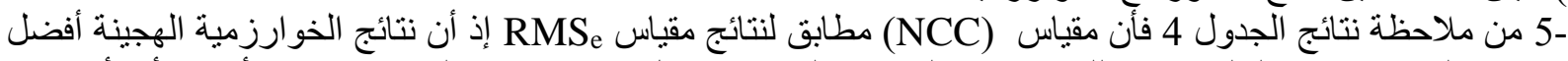

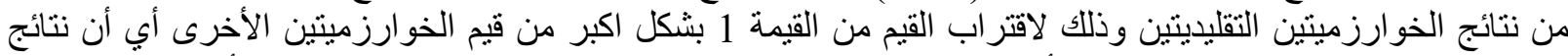

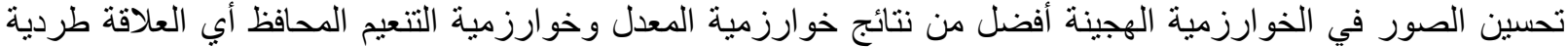
بشكل اكبر في نتائج الخو ارزمية الهجينة.

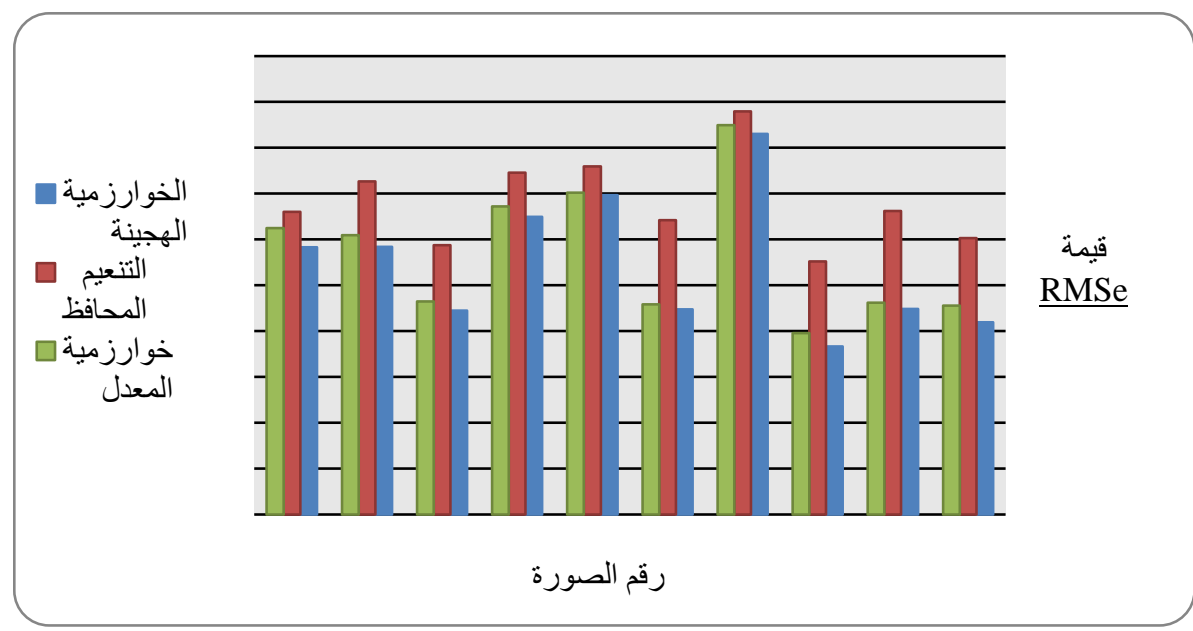

شكل رقم (7). رسم بياني يوضح مقدار الاختلاف للتحسين للخوارزميات عندما تكون الضوضاء نوع (salt \& pepper) بقيمة

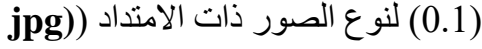




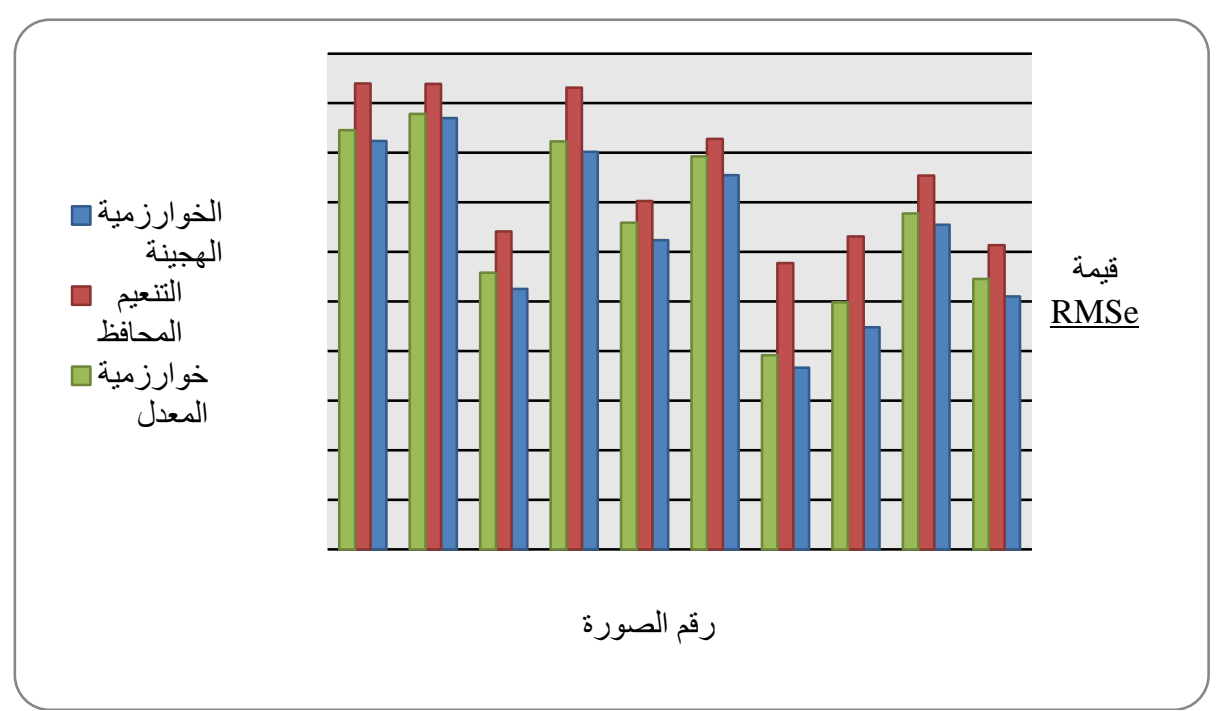

شكل رقم (8). رسم بياني يوضح مقدار الاختلاف للتحسين للخوارزميات عندما تكون الضوضاء نوع (salt \& pepper) بقيمة (0.1)

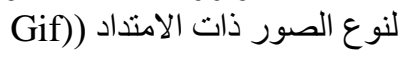

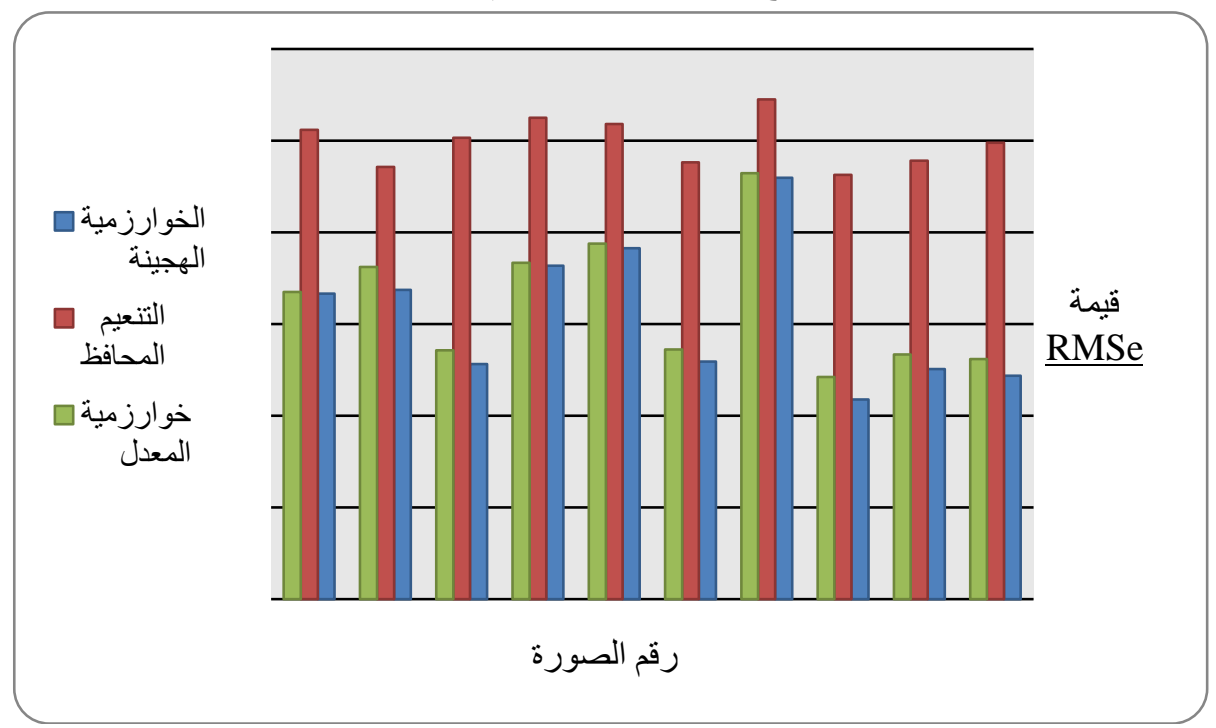

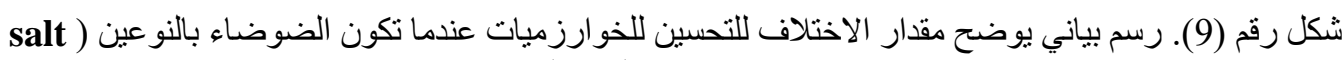

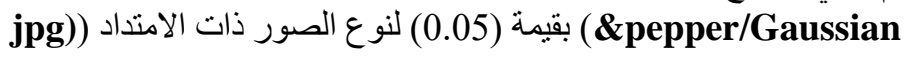




\begin{abstract}
المصادر
حامد علي, حنان, (2008) ,"خو ارزمية هجينة للتخلص من ضوضاء الصور", مجلة علوم الر افدين لعلوم الحاسبات [1] و الرياضيات, المجلد الخامس, العدد الأول, جامعة الموصل.
\end{abstract}

[2] Dmitrij Csetverikor, "Basic Algorithms for Digital Image Analysis" http://Visual.ipan.sztaki.hu./

[3] Gomes, J., and Velho L., (1997), "Image Processing for Computer Graphics" Translated by Silvio L., springer, inc.

[4] Gonzalez, Rafael E., (2002), "Digital Image Processing", 2nd edition, publisher: prentice-Hall.

[5] Jensen, R.J., (1982), "Introductory Digital Image Processing; A remote Sensing Perspective", Prentice-Hall.

[6] Katzenbeisser, S., and Petitcolas, F., (1999), "Information Hiding Techniques for Steganography and Digital Watermarking", Artech House, Boston London.

[7] Mather, Paul, M., (1987), "Computer Processing of Remotely Sensed Image" ,Johnand Sons, England.

[8] Robert, F.; Simon P., and Walker A., (2003), "Digital Filters" http://homepages.inf.ed.ac.uk/rbf/HIPR2/filtops.htm.

[9] Shannon, R., (2010), "Image File Formats"

http://www.yourhtmlsource.com/images/fileformats.html.

[10] Tinku, A., and Ajoy, K., (2005), "Image Processing Principles and Application", WILEY - Interscience USA.

[11] Umbaugh, Scott E., (1998), "Computer Vision and Image Processing a Practical Approach using CVIP Tools", Prentice Hall PTR.

[12] William, K., (2007), "Digital Image Processing", Fourth Edition, Wiley \& Sons. Inc. Publication. 


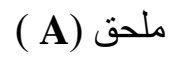

مجمو عة الصور التي تم استخدامها في البحث لبيان مدى تتو عها في الثنكل واللون و الحجم.

- 1.jpg نوع الصور
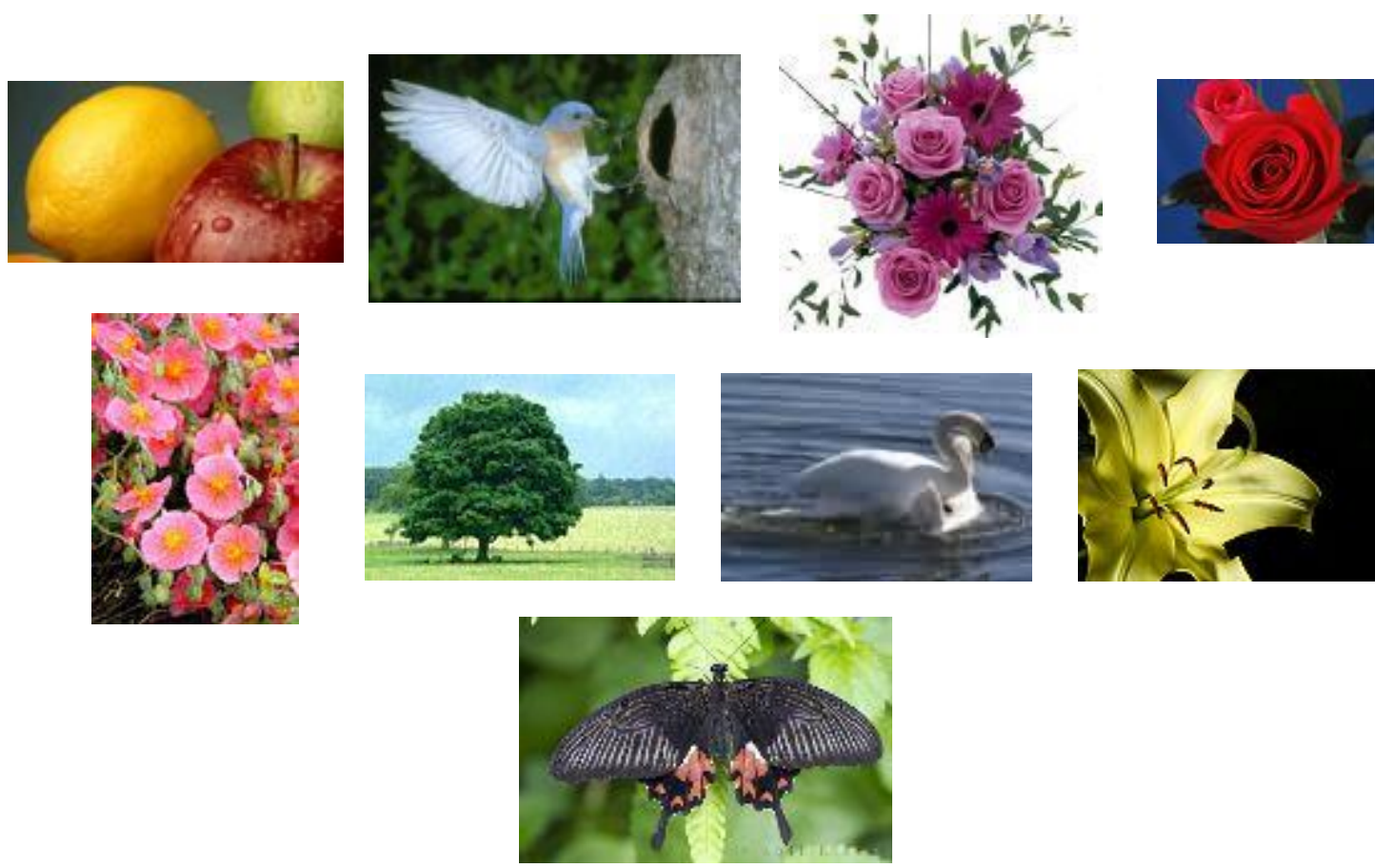

2-2 نوع الصور
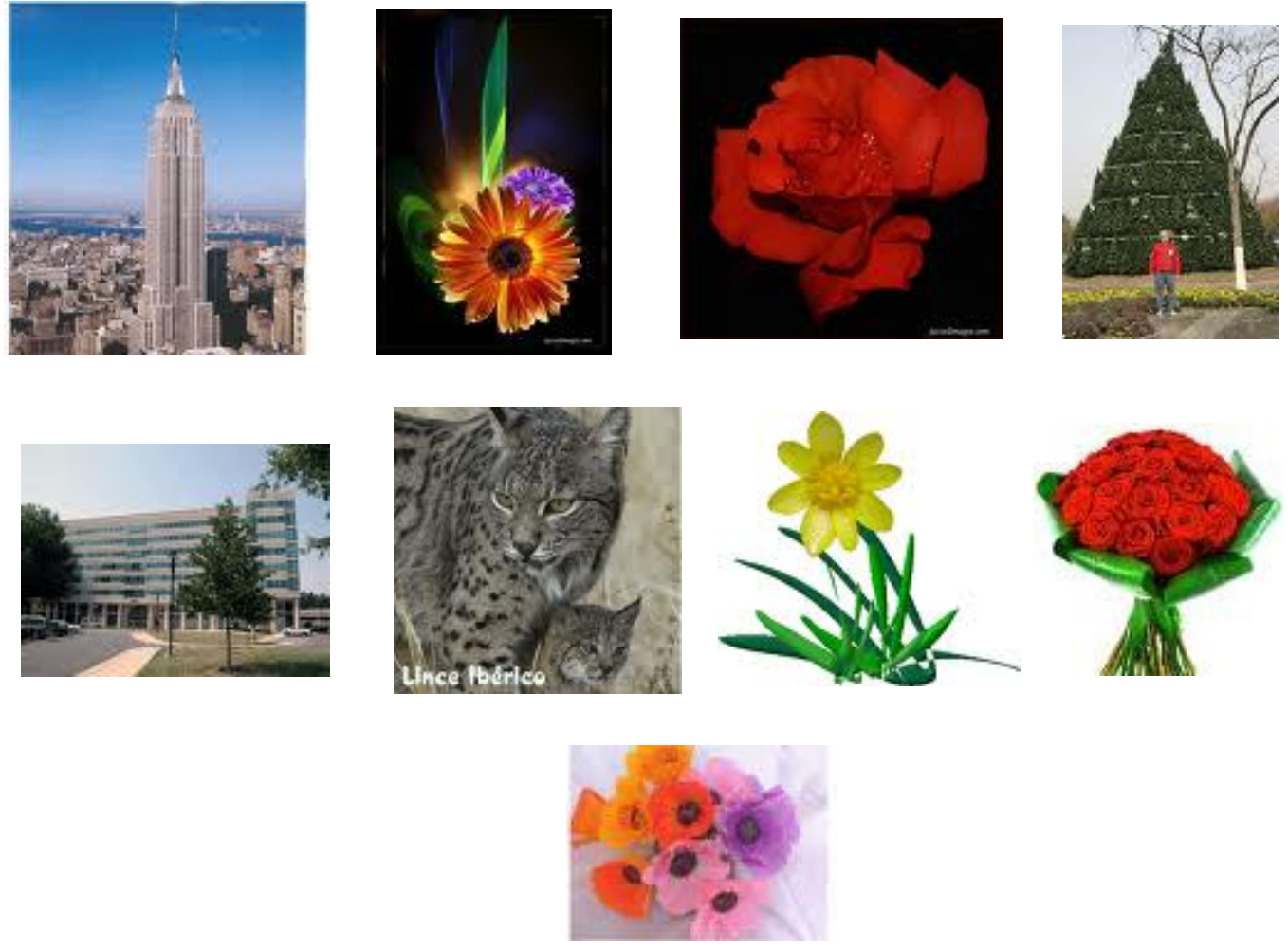